\title{
Does Student's Cognitive Flexibility Decrease During Pandemic? A New Approach to Measure Cognitive Flexibility
}

\author{
Hüseyin Zahid Cambaz ${ }^{1 *}$, Gülten Ünal ${ }^{1}$ \\ ${ }^{1}$ Ankara Yıldırım Beyazıt University, Psychology Department, Ankara, Turkey \\ e-mail: zahidcambaz@gmail.com; gunal@ybu.edu.tr
}

\begin{abstract}
The aim of this study is to examine the cognitive flexibility before and during the COVID-19 pandemic through a different performance measurement suitable for online application. In the study, three different data collected in 2019 (T19), 2020 (T20) and 2021 (T21) were compared with both within-and between-subject designs. One-hundred-and-twenty-three university students were included in the study for within-subjects, 239 for between-subject. According to the findings of the study, the cognitive flexibility of the students who continue formal education in T19 is higher than the students who continue distance education in T20 according to both within-subject and between-subject design. Besides, in both designs, fewer total words were produced in T19 than in T20. The sentences created by the students who continue their distance education in pandemic stay around less categories and contain more words. According to the within-subject findings obtained at T20 and T21, the cognitive flexibility and total words produced at T20 were greater. In other words, the scores of the students decreased over time during the pandemic period. In T21, cognitive flexibility is not related to vulnerability, perceived risk, and fear of COVID-19. However, according to the regression results, vulnerability at T21 was predicted by the total word and total emotional word in T20. Accordingly, producing more total words in T20 reduced vulnerability in T21; however, using more emotional words in T20 increased vulnerability in T21. In addition, the number of siblings was negatively associated with vulnerability. Lastly, cognitive flexibility test was found to be reliable.
\end{abstract}

Keywords: cognitive flexibility, performance measurement, emotional word, COVID-19, vulnerability, fear, perceived risk.

\section{Introduction}

Cognitive flexibility enables individuals to follow and succeed in complex tasks, and to adapt to changing demands. Besides, it is an important characteristic that depends on the interaction of sensorimotor and various cognitive mechanisms (lonescu, 2012). Cognitive flexibility is defined as the cognitive ability to switch between two different concepts and to think about different concepts at the same time (Scott, 1962). Therefore, cognitive flexibility is an executive function that involves shifting attention to another set (e.g., Lehto et al., 2003). Also, cognitive flexibility is defined as "the ability to benefit from different categories, the number of different categories used" in performance measures (e.g., Rietzschel, De Dreu and Nijstad 2007; Murray et. al, 1990). On the other hand, cognitive flexibility also reflects the revealing of unique semantic relationships (Jung-Beeman et al., 2004). Cognitive flexibility is a more complex process than a simple response shifting (Whiting et al., 2017). Neuroimaging studies have shown that when the language component is added to cognitive flexibility, more brain regions are activated in a complex manner. This is a complex process in which processing speed, working memory, and language processes have shared neural substrates with the frontal, parietal, and temporal regions of the brain (Barbey, Colom and Grafmanet, 2013).

The concept of cognitive flexibility is more evidence-based and larger than psychological flexibility (Whiting et al., 2017). Abstract definitions of psychological flexibility and cognitive flexibility are similar, but the functional goals of the concept of cognitive flexibility have reduced it to a shift setting (lonescu, 2012). Both concepts include a behavioural change as an intellectual or movement towards environmental changes. The significant difference between the two concepts is the role of the self and acceptance in the context. Accordingly, while cognitive flexibility includes adapting to changing cues, psychological flexibility includes seeing oneself apart from experiences and emotions and being able to accept these experiences

"Corresponding author: zahidcambaz@gmail.com 
instead of behavioural change and experiential avoidance when necessary (Whiting et al., 2017).

While psychological flexibility enables individuals to adapt to variable conditions, it also requires changing the behavioural repertoire when necessary (Kashdan and Rottenberg, 2010). It has been shown in different contexts that psychological flexibility is an important variable in maintaining mental health, and the lack of psychological flexibility in many psychopathology situations (Bond and Flaxman, 2006; Ciarrochi, Bilich and Godsellet, 2010; Kashdan and Rottenberg, 2010; McCracken and Morley, 2014). In times of such intense COVID-19 pandemic, both psychological and cognitive flexibility emerge as important concepts (e.g., Dawson and Golijani-Moghaddam, 2020; Fernandez Lynch et al., 2020; Kroska et al., 2020; Pakenham et al., 2020). Psychological flexibility has been shown to be effective in moderating the potentially harmful effects of COVID-19 risk factors (Pakenham et al., 2020). Besides, psychological flexibility is positively associated with well-being. It has been shown to be negatively associated with anxiety, depression, and distress related to COVID-19 (Dawson and Golijani-Moghaddam, 2020). Openness to experience, behavioural awareness, and valued action, which are components of psychological flexibility, might be important targets in intervention and prevention regarding COVID-19 (Kroska et al., 2020).

Apart from this, there are fewer studies in the context of cognitive flexibility than the concept of psychological flexibility. Jafari (2020) revealed that the self-reported cognitive flexibility taken with the Cognitive Flexibility Inventory was negatively correlated with anxiety during the COVID-19 period, and that the control sub-dimension of cognitive flexibility was negatively correlated with risk perception. Afshari, Hashemikamangar and Hashemikamangar (2021) found that both the control and alternatives sub-dimensions of cognitive flexibility are negatively correlated with the risk perception of COVID-19. Cognitive flexibility was found to have a negative correlation with social-distance fatigue, and a positive correlation with compliance with social distance (Seiter and Curran, 2021). Further, cognitive flexibility of COVID-19 patients who responded to home treatment was found to be significantly higher than those who did not respond to treatment (Jafari, 2020). As a result of these findings, it is understood that cognitive flexibility is an important variable in coping with COVID-19. However, all the studies presented here took measures of cognitive flexibility via self-report. In a period where cognitive flexibility is so important, it is crucial to conduct a study in which performance measurement is taken.

The current study examines cognitive flexibility in the context of students. The distance education process has started to take place in our lives more than ever due to COVID-19, and distance education has become inevitable due to current conditions (Kim, 2020). The pandemic has caused a global crisis in higher education (Raaper and Brown, 2020). According to Aucejo et al., (2020), 13\% of students post-poned their graduation due to COVID-19, 40\% lost their jobs, internships or job offers, and $29 \%$ expect less earnings by the age of 35 . Chen, Kaczmarek and Ohyamaet (2020) shows that according to the perception of a significant majority of students, burnout in distance education has increased and engagement in lessons has decreased. Fifteen percent of medical students are concerned that the pandemic will affect their specialty choices (Byrnes et al., 2020). Chinese students studying overseas stated that they have anxiety largely stemming from media discrimination and fear of COVID-19 (Ma and Miller, 2020). Besides these effects of the pandemic on students' academic and daily lives, there are also partially positive effects due to the transition to distance education. For example, students have faced many difficulties, their engagement has decreased, and their learning opportunities have been damaged, but there are also positive effects such as students' ability to attend classes in their location and flexibility in learning processes (Hill and Fitzgerald, 2020). Again, due to COVID-19, while 25\% of students' study hours increased by more than 4 hours per week, another $25 \%$ decreased by more than 5 hours per week (Aucejo et al., 2020). It is conceivable that COVID-19 reduces students' motivation, disrupts their daily routines, and reduces coping strategies required to deal with stressors (Grubic Badovinac and Johri, 2020). Therefore, it is important to study on and develop solutions for possible effects. In this context, it becomes important to understand the role of cognitive flexibility.

To measure cognitive flexibility, many methods have been used previously, including self-report and performance measurement. Some of the methods used in the performance measures of cognitive flexibility are Alternative Uses Test (AUT; Guilford, 1967), Torrance Creativity Test (TTCT; Almedia et al., 2008) and Wisconsin Card Sorting Test (WCST; Kongs et al., 2000). Cognitive flexibility is considered as a sub-dimension in creativity tests such as AUT and TTCT. Cognitive Flexibility Inventory (CFI; Dennis, and Vander Wal, 2010) and Cognitive Flexibility Scale (CFS; Bilgin, 2009) are among the most used scales in self-report measurement of cognitive flexibility. Studies investigating the relations of cognitive flexibility between self-report and performance measurement show that the correlation between selfreport and performance measures is either weak or absent, so it is suggested that neither measure the same structures or cognitive processes (Johnco, Wuthrich and Rapee, 2014). While problems such as social desirability (Webster, 2019) may arise in self-report measurements, a more reductionist approach 
is used in performance measures, so both approaches have their own advantages and disadvantages. Nevertheless, it is suggested that these two different approaches complement each other (Synder, Friedman and Hankin, 2020).

As a result, a different approach, used in Cambaz and Ünal's study (2021) and suitable for online application in this pandemic period, is used in the performance measurement of cognitive flexibility in this study. Like Barbey, Colom and Grafman (2013), this performance measurement includes processes to produce language. It is planned to compare this measurement taken at different times in university students, before and during the pandemic period, within-subject and between-subjects. Finally, it is aimed to examine the relationship of this cognitive flexibility with vulnerability, perceived risk, and fear in COVID-19.

\section{Hypotheses}

Hypothesis $1(\mathrm{H} 1)$ : The cognitive flexibility $(\mathrm{H} 1)$ and the total number of words produced (H1V2) of students at time T19 are expected to be higher than the levels of students at time T20.

Hypothesis $2(\mathrm{H} 2)$ : The cognitive flexibility $(\mathrm{H} 2 \mathrm{~V} 1)$ and the total number of words produced $(\mathrm{H} 2 \mathrm{~V} 2)$ measurements taken from the same students at T20 time are expected to be higher than the students at T21 time.

Hypothesis $3(\mathrm{H} 3)$ : There will be a negative relationship between cognitive flexibility and vulnerability, perceived risk and fear regarding COVID-19.

\section{Materials and Methods}

\section{Participants}

The data was collected at three different times (i.e., 2019, 2020, and 2021) from 239 students (207 women (86.6\%), for details see Table 1) studying at a state university in Ankara, Turkey. Ethical approval of the study was obtained from Ethics Committee. Participants were invited to the study in return for additional points for the course.

\section{Table 1}

Number of participants

\begin{tabular}{ccc}
\hline & \multicolumn{2}{c}{ Design } \\
\cline { 2 - 3 } Year & Between-subject & Within-Subject \\
\hline 2019 & 100 & 16 \\
2020 & 139 & 123 \\
2021 & - & 123 \\
& Total: 239 & Total:123 \\
\hline
\end{tabular}

\section{Design}

\section{Dependent variable}

Cognitive flexibility and produced total words (collected in T19, T20, and T21), and vulnerability, perceived risk, and fear of COVID-19 (collected only in T21) were the dependent variables of the study.

\section{Independent variables}

Within-Subject. Pre-pandemic (T19), pandemic (T20) and second pandemic (T21) data obtained from the same participants at different times were compared. The within-subject results were obtained by comparing T19 with T20, and T20 with T21.

Between-Subjects. Before and after pandemic: 2 (scores before pandemic [T19] x scores during pandemic [T20]) 


\section{Measurements \\ Cognitive flexibility task}

The performance measurement in Cambaz and Ünal's (2021) study was used for assessing cognitive flexibility. The operational definition of cognitive flexibility in this measure is "the ability to benefit from different categories, the number of different categories used, revealing unique semantic relationships" (e.g., Jung-Beeman et al., 2004; Murray et. al, 1990; Rietzschel, De Dreu and Nijstadet, 2007). A total of 40 words from 8 different categories (e.g., school, furniture, emotions), 5 from each category (e.g., student, desk, happy), were presented in a table. The categories used in the table are predetermined and the words from these categories presented as mixed in this table. Thus, an objective approach was demonstrated in determining the different categories used by the participants. The instruction given to the participants is "Please form five different sentences using at least one of the above words, or as many as you want." The cognitive flexibility score of the participants was obtained through the following formula.

$$
\sum_{n=1}^{5} k_{n}-1
$$

\section{$k=$ number of different categories used in a sentence}

Using one category in a sentence does not generate points because the participants have already been asked to use at least one word. For example, "I feel unhappy." cognitive flexibility score was considered 0 for the sentence. Because in this sentence, only one word from one category was used, and the instructions given to the participants were already stated for them to use at least one word. All participants, without exception, used at least one word in each sentence. For instance, considering the sentence "The cat and dog were playing happily.", three different words from two different categories are used and the cognitive flexibility score for this sentence is 1 . Words belonging to the same category used in the same sentence do not produce cognitive flexibility scores. Cognitive flexibility score was calculated as "the number of different categories used in a sentence - 1" within this framework. The total score is obtained by adding the flexibility score generated for all sentences. As a result, cognitive flexibility here is not the use of a word given in the table in a sentence, but the ability of the participants to combine words in different categories, which are more difficult to use together.

\section{COVID-19 vulnerability, perceived risk, and fear}

As in Yıldırım, Geçer and Akgül's (2021) study, vulnerability, perceived risk, and fear measurements were also collected. For vulnerability, the following question was asked to the participants: "In your opinion, what is the probability of developing coronavirus disease during the coronavirus outbreak?". For perceived risk, the participants asked the following question: "Compared to most people your age and sex, what would you say your chances are for developing novel coronavirus?". Lastly, "I am afraid of novel coronavirus" and "I am terrified by a novel coronavirus" was used for fear measurement. All questions were 5-point Likert type, and high scoring for each question indicated higher vulnerability, higher perceived risk, and higher fear, respectively.

\section{Procedure}

Participants were invited to the research via the online form. Participants who approved the consent form first answered the cognitive flexibility task, then the COVID-19 questions (T21) and the demographic form $(\mathrm{T} 19, \mathrm{~T} 20)$.

\section{Results}

\section{Reliability of the Measurement}

To test the reliability of cognitive flexibility for five different sentences in the T19 data, the Cronbach alpha internal consistency coefficient was examined. The result was .78. The internal consistency coefficient was found as .77 and .73 for the T20 and T21, respectively. To measure the test-retest reliability, the correlation between T20 and T21 data from the same participants was examined. Accordingly, the test-retest correlation coefficient was found to be .64 . 


\section{Within-Subject Results}

The analysis of the data was performed by using the SPSS v23 statistical analysis program. Below and above 2.5 standard deviations were excluded from the analysis. Paired-sample t-test analysis' results indicated a significant difference in the cognitive flexibility scores between T20 measurement $(M=3.96$, $S D=2.82)$ and the T21 measurement $(M=3.31, S D=2.55)$ conditions; $t(123)=3.17, p=.00$. There was a significant difference in the total words scores for between T20 measurement $(M=41.87, S D=9.77)$ and the T21 measurement $(M=39.02, S D=7.75)$ conditions; $t(123)=3.54, p<.001$.

There was a difference between the cognitive flexibility scores for between T19 measurement $(M=$ $5.50, S D=1.91)$ and T20 measurement $(M=4.43, S D=2.71)$ for same participants ( $t(13)=1.10, p=.29)$ was not significant. However, the total words' scores for T19 measurement $(M=32.86, S D=11.65)$ and for T20 measurement $(M=46.21, S D=10.37)$ for same participants $(t(13)=4.13, p<.001)$ was significant.

\section{Between-Subjects Results}

The MANOVA test was applied to examine the cognitive flexibility and total word differences of different students whose data were collected at T19 and T20 (gender and age were controlled). According to Box's Covariance Equality Test results $(F(3,3431724)=2.01, p=.11)$, it was found that the covariance equality assumption was met. According to Levene's Equality of Variance Test results, it was found that the equality of variance assumption was met for cognitive flexibility $F(1,237)=, 007, p=.94)$ and total words $(F(1,237)=.38, p=.54)$.

The different measurement times (T19-T20) had a significant effect in multivariate test $(\Lambda=.819$, $F(2,234)=25.84$. Namely, T19 measurement of cognitive flexibility $(N=99, M=4.97, S D=3.01)$ was significantly higher than T20 measurement $(N=140, M=3.76, S D=2.66) ; F(1,235)=10.10, p=.00$, $\eta 2\urcorner=.04$. Also, $T 19$ measurement of total words $(N=99, M=34.51, S D=8.99)$ was significantly lower than T20 measurement $(N=140, M=41.89, S D=9.83) ; F(1,235)=34.10, p=.00, \eta 2\urcorner=.12$. In other words, students who continued their education with formal education in 2019 used significantly more categories (their flexibility was higher) but they used significantly less words. Gender and age did not cause a significant difference.

\section{Correlations Among Variables}

There is a correlation of .64 between T20 and T21 time of cognitive flexibility, and a correlation of .52 between T20 and T21 times of the total word (see Table 2). There is a correlation of .37 between vulnerability and perceived risk; .23 between vulnerability and cognitive flexibility in T20; -.23 between vulnerability and the total words in T20; and .23 between vulnerability total emotional words in T20. No correlation was found for cognitive flexibility and total word data at T21 time obtained simultaneously with COVID-19 related variables. To examine how the data explains vulnerability, regression analysis was performed.

Table 2

Correlation coefficients

\begin{tabular}{|c|c|c|c|c|c|c|c|c|c|c|c|c|}
\hline & 1 & 2 & 3 & 4 & 5 & 6 & 7 & 8 & 9 & 10 & 11 & 12 \\
\hline 1.CF-T20 & 1 & & & & & & & & & & & \\
\hline 2.CF-T21 & $.64^{* \star}$ & 1 & & & & & & & & & & \\
\hline 3.TW-T20 & .08 & -.05 & 1 & & & & & & & & & \\
\hline 4. TW-T21 & .08 & .00 & $.52^{\star \star}$ & 1 & & & & & & & & \\
\hline 5. TEW-T20 & $.50^{\star \star}$ & $.30^{\star \star}$ & $.18^{\star}$ & .04 & 1 & & & & & & & \\
\hline 6. TEW-T21 & $26^{\star \star}$ & $.42^{\star \star}$ & -.07 & -.04 & $.39^{\star \star}$ & 1 & & & & & & \\
\hline 7. Vulnerability & $.23^{\star}$ & .13 & $-.23^{*}$ & -.10 & $23^{* \star}$ & .08 & 1 & & & & & \\
\hline 8. P. Risk & -.04 & -.04 & -.20 & -.09 & -.03 & .09 & $37^{\star \star}$ & 1 & & & & \\
\hline 9. Fear & -.10 & -.03 & -.17 & -.02 & -.14 & -.04 & .11 & .14 & 1 & & & \\
\hline 10. P. SEL & -.06 & -.09 & -.12 & -.16 & -.03 & .17 & -.05 & -.01 & .05 & 1 & & \\
\hline 11. Siblings & -.01 & -.06 & .04 & .04 & .04 & -.01 & .10 & -.03 & $-.25^{\star \star}$ & -.16 & 1 & \\
\hline 12. Age & -.06 & -.05 & -.08 & -.16 & -.08 & -.04 & .13 & .01 & .03 & -.10 & .02 & 1 \\
\hline
\end{tabular}




\section{Regression for COVID-19 Vulnerability}

The model in Table 3 explains $31 \%$ of the vulnerability variance, $F(11,112)=4.67, p=.00$ (see Table 3 ). Accordingly, the perceived risk $(\beta=.348, t=4.28, p=.00)$ predicts vulnerability. Cognitive flexibility, total word, and total emotional words obtained at the same time (in T21) with the perceived risk variable did not predict vulnerability. Surprisingly, the total word $(\beta=-.22, t=-2.311, p=.02)$ and total emotional words $(\beta=.24, t=2.48, p=.02$ ) obtained from the same participants a month ago (in T20) predicts vulnerability. In other words, performance measurements taken from the participants simultaneously with the vulnerability measurement did not predict vulnerability. Performance measurements (total word and total emotional word) collected a month ago predicted vulnerability. Accordingly, while the total word collected three weeks ago seems to decrease the upcoming perceived vulnerability, the total emotional word used a month ago seems to increase vulnerability.

Table 3

The model for vulnerability of COVID-19

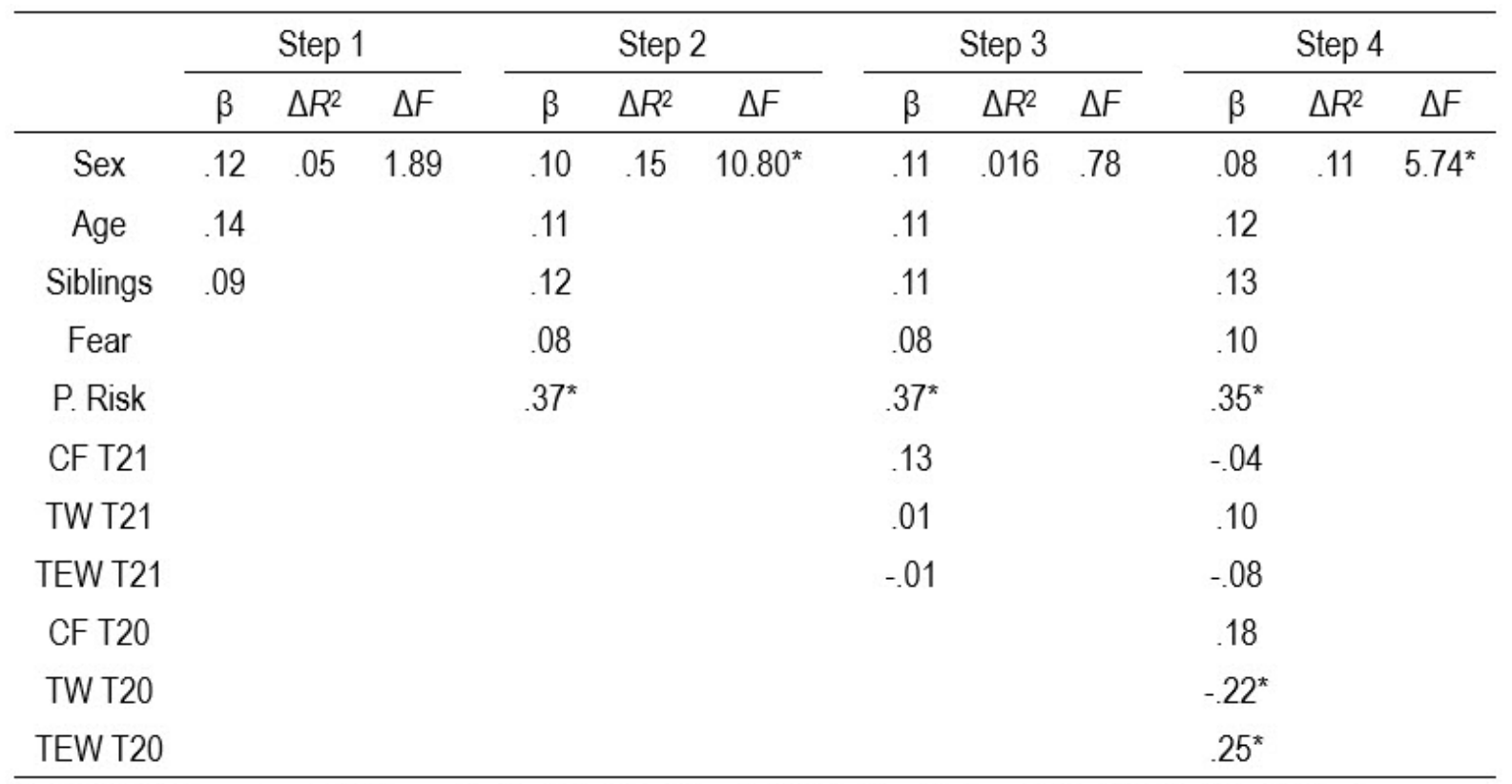

*. $p<0.05$, P. Risk $=$ perceived risk, $C F=$ cognitive Flexibility, $T W=$ total word, $T E W=$ total emotional word, $T 20=2020$ data, $T 21=2021$ data

\section{Discussion}

In this study, a performance measure was used benefiting from the language production processes of cognitive flexibility. This performance measure is also in line with the definition of cognitive flexibility regarding usage of unique semantic relationships (Jung-Beeman et al., 2004). It is understood that this performance measurement, suitable for online application during this pandemic period, gives reliable results. According to Hulin, Netemeyer and Cudeck (2001), 0.6-0.7 levels are acceptable, so although it is a performance measurement, it is understood that the internal consistency coefficient and test-retest coefficient are sufficiently reliable. This test-retest coefficient is even higher than the Turkish version of the Cognitive Flexibility Inventory (Gülüm and Dağ, 2012), which is a self-report measure. Some of the disadvantages mentioned about performance measures are that they require a lot of time and money and their investment in maximal performance in the artificial environment (Coman and Richardson, 2006). However, the current proposed measurement lacks these disadvantages and participants do not have to strain for a maximum performance. So, the flexibility score also depends on a desire that the participants are not aware of. In creativity tests such as AUT and TTCT, the responses of the participants are obtained first, and then the coders try to obtain how many different categories those answers belong to, and with a relatively subjective approach. In this proposed method, the cognitive flexibility score is evaluated within the framework of objective criteria in the sentences.

According to the between-subject findings of the study, the cognitive flexibility of students who continue formal education in 2019 (T19) is higher than that of students who continue distance education in 2020 (T20), thus H1V1 is confirmed. However, the total words produced by students attending formal 
education in 2019 is lower than the students who continue distance education in 2020, thus H1V2 is confirmed just the opposite way. In other words, while students in 2019 formed sentences with fewer words around more categories, students in 2020 formed more words with less categories. This finding was confirmed by the within-subject results comparing the T19 and T20 results, where a limited participant was available. In T19 time, the participants produced sentences with fewer words around more categories, compared to the T20. Both within-subject and between-subject research results reveal a pattern like this: students who continued their distance education during the pandemic period used more words around fewer categories, that is, they were stuck around certain categories. This could be considered as a reflection of less cognitive flexibility.

According to the within-subject findings at T20 and T21 times, students produced more cognitive flexibility and total words at $\mathrm{T} 20$ than at $\mathrm{T} 21$, thus $\mathrm{H} 2 \mathrm{~V} 1$ and $\mathrm{H} 2 \mathrm{~V} 2$ is confirmed. One reason for the cognitive flexibility and total word in T20 time is higher than at T21 time may be that the approaching exams in T21 time plays a role as a stressor, since high anxiety is associated with low cognitive flexibility (e.g., Kalia, Knauft and Hayatbiniet 2020; Park and Moghaddam, 2017; Yu, Yu and Lin, 2020). Exam anxiety during the pandemic period may have caused worse results. Although the data were collected at the same time, no correlation was found between the cognitive flexibility, total word, total emotional used, and COVID-19 variables at T21 time, thus H3 is not confirmed. Also, no correlation was found between perceived risk of COVID-19 and self-reported overall cognitive flexibility (Kalia, Knauft and Hayatbiniet 2020). Surprisingly, however, regression analysis revealed that vulnerability in T21 was predicted by the total word and total emotional word in T20. The high number of total words in T20 time decreased vulnerability, and the high number of total emotional words in T20 time increased vulnerability in T21. This may mean that expressing ourselves during the pandemic period reduces our vulnerability, but when emotional expression is involved, our vulnerability increases. The study also found that the number of siblings was negatively correlated with the fear of COVID-19. According to the findings of Ma and Miller (2020), perceived social support in students during the pandemic period is negatively related to anxiety and stress. Greater number of siblings may be related with greater perceived social support.

Cognitive flexibility is associated with positive outcomes during the pandemic period (e.g., Afshari, Hashemikamangar and Hashemikamangar, 2021; Kalia, Knauft and Hayatbiniet 2020; Seiter and Curran, 2021). In the current study, obviously, distance education period plays an important role in reducing students' cognitive flexibility. In addition, stressors such as exam stress could further reduce cognitive flexibility. If conditions such as the loss of a relative are added to this picture, it may be even more difficult to cope with this situation. Therefore, some strategies should be followed to increase the cognitive flexibility of students and make them to adapt to the distance education process in a better way.

The first strategy may be as follows: Social interactions of individuals -within the scope of pandemic possibilities- allow healthy progress. In the current study, it has been shown that the number of siblings is negatively related to the perceived fear of COVID-19. In addition, the vulnerability of the participants who expressed themselves more (producing more total words) after one month was found to be lower. However, the vulnerability of the participants who expressed themselves around emotional words was higher after one month. Therefore, it seems important to provide environments where participants can express themselves without experiencing excessive emotional intensity. In this period, students' access to mental health services should be facilitated, albeit virtual, and social assistance should be provided to students in special situations (Liu et al., 2020). Online education is proposed to include planning, implementation and reflection stages and there are tasks to be completed in these stages (Kim, 2020). Kim suggests that even if the transition to normal classroom teaching is difficult in the current situation, the environment, and activities like the classroom environment should be provided in online teaching and important components such as creativity, critical thinking, and communication should be presented to students. Students may have some suggestions to make the lessons more efficient (Chen, Kaczmarek and Ohyama, 2020). Working with small groups of previously distributed cases could be more beneficial for students than lecture alone. Interactive lessons are also more beneficial and ungraded quizzes can be made useful at this stage so that students can be integrated into the lesson. Interactive teaching style is the main predictor of student satisfaction and effectiveness of teaching (Fatani, 2020). Students may improve their metacognitive skills (e.g., cognitive flexibility) to better adapt to education in COVID-19 (Qadir and AlFuqaha, 2020). Qadir and Al-Fuqaha suggest that it may be beneficial for students to have a basic knowledge on the functioning of memory, the connection of neurons with each other, how to learn best and how to consolidate information to improve their meta-cognitive processes.

One limitation of this study is the small number of participants for the within-subject design at T19 and T20 time. In addition, the proposed novel method needs to be studied in different contexts and variables. Not only the quantity but also the quality of the sentences produced by the participants around 
certain words may provide meaningful data. Different qualitative structures may occur in the content of sentences according to the number of different categories used. Therefore, qualitative analysis and semantical evaluation of the generated sentences might provide new perspectives. Also, for future studies, it may be suggested to investigate the relationship between different performance measures of cognitive flexibility and the currently proposed performance measurement. While distance education extended the study time of some students, it decreased some, as well (Aucejo, 2020). Examining personality types of participants (e.g., extroverted, or introverted) might help to explain these seemingly contradictory results.

\section{Conclusion}

In this study, a novel approach was used to measure cognitive flexibility. The correlation between the measurements collected at different times points to a disposition, which could be called as trait, for the number of word production and the use of words from different categories. As a result, in a period when the COVID-19 pandemic was experienced so intensively, students seem to get heavily affected both cognitively and emotionally. Current research reveals that cognitive flexibility, which is a metacognitive process, is affected by this process, as well. Accordingly, in this period, students preferred to produce many words around fewer categories, indicating low cognitive flexibility, rather than producing their semantic resources efficiently. However, performance measures such as producing many words or focusing on emotional words as a general dispositional strategy have also been found to predict future vulnerability to COVID-19. More research is needed to examine important psychological resources such as cognitive flexibility that may be affected by this period, and to develop strategies to protect these resources.

\section{Acknowledgements}

The authors are grateful to Dr. Emine İnan who helped in the data collection process and to all participants of the study, and to the members of Social Cognition Psychology Laboratory for always being there from the very beginning.

\section{Conflict of interests}

The authors declare no conflict of interest.

\section{References}

Afshari, A., Hashemikamangar, S., \& Hashemikamangar, S. S. (2021). The correlation of perceived stress and professional concerns during COVID-19 pandemic among Iranian dentists: the mediating role of cognitive flexibility. Dentistry 3000 , 9(1). https://doi.org/10.5195/d3000.2021.119

Almeida, L. S., Prieto, L. P., Ferrando, M., Oliveira, E., ve Ferrándiz, C. (2008). Torrance Test of Creative Thinking: The Question of Its Construct Validity. Thinking Skills and Creativity, 3(1), 53-58. https://doi.org/10.1016/j.tsc.2008.03.003

Aucejo, E. M., French, J., Araya, M. P. U., \& Zafar, B. (2020). The impact of COVID-19 on student experiences and expectations: Evidence from a survey. Journal of Oublic Economics, 191, 104271. https://doi.org/10.1016/j.jpubeco.2020.104271

Barbey, A. K., Colom, R., \& Grafman, J. (2013). Architecture of cognitive flexibility revealed by lesion mapping. Neuroimage, 82, 547-554. https://doi.org/10.1016/j.neuroimage.2013.05.087

Bilgin, M. (2009). Developing a cognitive flexibility scale: Validity and reliability studies. Social Behavior and Personality: An International Journal, 37(3), 343-353. https://doi.org/10.2224/sbp.2009.37.3.343

Bond, F. W., \& Flaxman, P. E. (2006). The ability of psychological flexibility and job control to predict learning, job performance, and mental health. Journal of Organizational Behavior Management, 26(1-2), 113-130. https://doi.org/10.1300/ J075v26n01_05

Byrnes, Y. M., Civantos, A. M., Go, B. C., McWilliams, T. L., \& Rajasekaran, K. (2020). Effect of the COVID-19 pandemic on medical student career perceptions: a national survey study. Medical Education Online, 25(1), 1798088. https://doi.or g/10.1080/10872981.2020.1798088

Cambaz, H. Z., \& Ünal, G. (2021). The Effect of Abstract and Concrete Boundaries on Cognitive Flexibility. Hacettepe University Journal of Faculty of Letters. https://doi.org/10.32600/huefd.772811

Chen, E., Kaczmarek, K., \& Ohyama, H. (2020). Student perceptions of distance learning strategies during COVID-19. Journal of dental education. https://doi.org/10.1002/jdd.12339

Ciarrochi, J., Bilich, L., \& Godsell, C. (2010). Psychological flexibility as a mechanism of change in acceptance and commitment therapy. In R. A. Baer (Ed.), Assessing mindfulness and acceptance processes in clients: Illuminating the theory and practice of change (p. 51-75). Context Press/New Harbinger Publications.

Coman, L., \& Richardson, J. (2006). Relationship between self-report and performance measures of function: a systematic review. Canadian Journal on Aging/La Revue Canadienne du Vieillissement, 25(3), 253-270. Retrieved from https:// www.muse.jhu.edu/article/208987

Dawson, D. L., \& Golijani-Moghaddam, N. (2020). COVID-19: Psychological flexibility, coping, mental health, and wellbeing 
Cambaz, Z. H. \& Ünal, G. (2021). Does student's cognitive flexibility decrease during pandemic? A new approach to measure cognitive flexibility, International Journal of Cognitive Research in Science, Engineering and Education (IJCRSEE), 9(1), $13-22$.

in the UK during the pandemic. Journal of Contextual Behavioral Science, 17, 126-134. https://doi.org/10.1016/j. jcbs.2020.07.010

Dennis, J. P., \& Vander Wal, J. S. (2010). The cognitive flexibility inventory: Instrument development and estimates of reliability and validity. Cognitive Therapy and Research, 34(3), 241-253. https://doi.org/10.1007/s10608-009-9276-4

Fernandez Lynch, H., Dickert, N. W., Zettler, P. J., Joffe, S., \& Largent, E. A. (2020). Regulatory flexibility for COVID-19 research. Journal of Law and the Biosciences, 7(1), Isaa057. https://doi.org/10.1093/jlb/lsaa057

Fatani, T. H. (2020). Student satisfaction with videoconferencing teaching quality during the COVID-19 pandemic. BMC Medical Education, 20(1), 1-8. https://doi.org/10.1186/s12909-020-02310-2

Grubic, N., Badovinac, S., \& Johri, A. M. (2020). Student mental health in the midst of the COVID-19 pandemic: A call for further research and immediate solutions. International Journal of Social Psychiatry, 66(5), 517-518. https://doi. org/10.1177/0020764020925108

Guilford, J. P. (1967). The nature of human intelligence. New York: McGraw-Hill

Gülüm, I. V., \& Dağ, I. (2012). The Turkish adaptation, validity and reliability study of the Repetitive Thinking Questionnaire and the Cognitive Flexibility Inventory. Anatolian Journal of Psychiatry, 13(3).

Hill, K., \& Fitzgerald, R. (2020). Student perspectives of the impact of COVID-19 on learning. All Ireland Journal of Higher Education, 12(2).

Hulin, C., Netemeyer, R., \& Cudeck, R. (2001). Can a reliability coefficient be too high? Journal of Consumer Psychology, $55-58$.

Ionescu, T. (2012). Exploring the nature of cognitive flexibility. New Ideas in Psychology, 30(2), 190-200. https://doi.org/10.1016/j. newideapsych.2011.11.001

Jafari, A. (2020). Comparing Cognitive Flexibility, Psychological Capital and Coping Strategies with Pain between Individuals with COVID-19 Responding and Non-Responding to Home Treatment. Journal of Counseling Research, 19(74), 4-35. https://doi.org/10.29252/jcr.19.74.4

Johnco, C., Wuthrich, V. M., \& Rapee, R. M. (2014). Reliability and validity of two self-report measures of cognitive flexibility. Psychological Assessment, 26(4), 1381. https://doi.org/10.1037/a0038009

Jung-Beeman, M., Bowden, E. M., Haberman, J., Frymiare, J. L., Arambel-Liu, S., Greenblatt, R., ... \& Kounios, J. (2004). Neural activity when people solve verbal problems with insight. PLoS Biol, 2(4), e97. https://doi.org/10.1371/journal. pbio.0020097

Kalia, V., Knauft, K., \& Hayatbini, N. (2020). Cognitive flexibility and perceived threat from COVID-19 mediate the relationship between childhood maltreatment and state anxiety. PloS One, 15(12), e0243881. https://doi.org/10.1371/journal. pone.0243881

Kashdan, T. B., \& Rottenberg, J. (2010). Psychological flexibility as a fundamental aspect of health. Clinical Psychology Review, 30(7), 865-878. https://doi.org/10.1016/j.cpr.2010.03.001

Kim, J. (2020). Learning and teaching online during Covid-19: Experiences of student teachers in an early childhood education practicum. International Journal of Early Childhood, 52(2), 145-158. https://doi.org/10.1007/s13158-020-00272-6

Kongs, K.S., Thompson, L.L., Iverson, G.L. ve Heaton, R.K. (2000). Wisconsin card sorting test-64 card version (WCST-64). Psychological Assessment Resources, Odessa.

Kroska, E. B., Roche, A. I., Adamowicz, J. L., \& Stegall, M. S. (2020). Psychological flexibility in the context of COVID-19 adversity: Associations with distress. Journal of Contextual Behavioral Science, 18, 28-33. https://doi.org/10.1016/j. jcbs.2020.07.011

Lehto, J. E., Juujärvi, P., Kooistra, L., \& Pulkkinen, L. (2003). Dimensions of executive functioning: Evidence from children. British Journal of Developmental Psychology, 21(1), 59-80. https://doi.org/10.1348/026151003321164627

Liu, C. H., Pinder-Amaker, S., Hahm, H. C., \& Chen, J. A. (2020). Priorities for addressing the impact of the COVID-19 pandemic on college student mental health. Journal of American College Health, 1-3. https://doi.org/10.1080/074484 81.2020 .1803882

Ma, H., \& Miller, C. (2020). Trapped in a double bind: Chinese overseas student anxiety during the COVID-19 pandemic. Health Communication, 1-8. https://doi.org/10.1080/10410236.2020.1775439

Matheson, G. J. (2019). We need to talk about reliability: making better use of test-retest studies for study design and interpretation. PeerJ, 7, e6918. https://doi.org/10.7717/peerj.6918

McCracken, L. M., \& Morley, S. (2014). The psychological flexibility model: a basis for integration and progress in psychological approaches to chronic pain management. The Journal of Pain, 15(3), 221-234. https://doi.org/10.1016/j. pain.2013.10.014

Murray, N., Sujan, H., Hirt, E. R., \& Sujan, M. (1990). The influence of mood on categorization: A cognitive flexibility interpretation. Journal of Personality and Social Psychology, 59(3), 411. https://doi.org/10.1037/0022-3514.59.3.411

Pakenham, K. I., Landi, G., Boccolini, G., Furlani, A., Grandi, S., \& Tossani, E. (2020). The moderating roles of psychological flexibility and inflexibility on the mental health impacts of COVID-19 pandemic and lockdown in Italy. Journal of Contextual Behavioral Science, 17, 109-118. https://doi.org/10.1016/j.jcbs.2020.07.003

Park, J., \& Moghaddam, B. (2017). Impact of anxiety on prefrontal cortex encoding of cognitive flexibility. Neuroscience, 345 , 193-202. https://doi.org/10.1016/j.neuroscience.2016.06.013

Qadir, J., \& Al-Fuqaha, A. (2020). A student primer on how to thrive in post-COVID-19 engineering education.

Raaper, R., \& Brown, C. (2020). The Covid-19 pandemic and the dissolution of the university campus: Implications for student support practice. Journal of Professional Capital and Community. https://doi.org/10.1108/JPCC-06-2020-0032

Rietzschel, E. F., De Dreu, C. K., \& Nijstad, B. A. (2007). Personal need for structure and creative performance: The moderating influence of fear of invalidity. Personality and Social Psychology Bulletin, 33(6), 855-866. https://doi. org/10.1177/0146167207301017

Scott, W. A. (1962). Cognitive complexity and cognitive flexibility. Sociometry, 405-414. https://doi.org/10.2307/2785779

Seiter, J. S., \& Curran, T. (2021). Social-distancing fatigue during the COVID-19 pandemic: a mediation analysis of cognitive flexibility, fatigue, depression, and adherence to CDC guidelines. Communication Research Reports, 1-11. https://doi. org/10.1080/08824096.2021.1880385

Snyder, H. R., Friedman, N. P., \& Hankin, B. L. (2020). Associations Between Task Performance and Self-Report 
Measures of Cognitive Control: Shared Versus Distinct Abilities. Assessment, 1073191120965694. https://doi. org/10.1177/1073191120965694

Webster, J. D. (2019). Self-report wisdom measures: Strengths, limitations, and future directions. In R. J. Sternberg \& J. Glück (Eds.), The Cambridge handbook of wisdom (p. 297-320). Cambridge University Press. https://doi. org/10.1017/9781108568272.015

Whiting, D. L., Deane, F. P., Simpson, G. K., McLeod, H. J., \& Ciarrochi, J. (2017). Cognitive and psychological flexibility after a traumatic brain injury and the implications for treatment in acceptance-based therapies: A conceptual review. Neuropsychological Rehabilitation, 27(2), 263-299. https://doi.org/10.1080/09602011.2015.1062115

Yıldırım, M., Geçer, E., \& Akgül, Ö. (2021). The impacts of vulnerability, perceived risk, and fear on preventive behaviours against COVID-19. Psychology, Health \& Medicine, 26(1), 35-43. https://doi.org/10.1080/13548506.2020.1776891

Yu, Y., Yu, Y., \& Lin, Y. (2020). Anxiety and depression aggravate impulsiveness: the mediating and moderating role of cognitive flexibility. Psychology, Health \& Medicine, 25(1), 25-36. https://doi.org/10.1080/13548506.2019.1601748 